\title{
Revisão sistemática sobre os efeitos dos probióticos na depressão e ansiedade: terapêutica alternativa?
}

\author{
A systematic review of the effects of probiotics on depression \\ and anxiety: an alternative therapy?
}

Miryam de Souza Minayo (https://orcid.org/0000-0003-3521-3113) ${ }^{1}$

Iasmim Miranda (https://orcid.org/0000-0002-5742-5371) ${ }^{2}$

Raquel Senna Telhado (https://orcid.org/0000-0002-9136-3493) ${ }^{2}$

${ }^{1}$ Cooperação Internacional, Fundação Oswaldo Cruz. Av. Brasil 4.365 (prédio atrás do castelo), Manguinhos. 21040-360 Rio de Janeiro RJ Brasil.

miryam.minayo@fiocruz.br

${ }^{2}$ Centro Universitário IBMR, Laureate

International Universities. Rio de Janeiro RJ Brasil.

\begin{abstract}
This review aims to understand and analyse the effects of probiotics on depression, anxiety and psychological stress. These disorders are among the leading causes of disability worldwide. Conventional pharmacotherapies usually have a poor response or adverse side effects. In this context, recent studies have demonstrated a dense bi-directional communication named gut-brain axis. Evidences are demonstrating the relationship between disturbance in the enteric microbiome and psychiatric disorders, paving the way for the emergence of alternative therapies. A systematic search for randomized double/triple blind placebo-controlled clinical trials was performed in PubMed, Scopus and Lilacs. The studies selection followed the recommendations of the main items for report systematic reviews and meta-analyses (PRISMA). Nine articles met the criteria and were analysed for effects on depression, anxiety, psychological stress and biomarkers. Seven fou$n d$ positive results in at least one of the items. We concluded that the use of probiotics to alleviate depressive symptoms and anxiety is promising, mainly due to its potential anti-inflammatory effect, but additional and more rigorous double blind randomized clinical trials are necessary to endorse such conclusions.
\end{abstract}

Key words Depression, Anxiety, Probiotics, Microbiota, Gut-brain axis
Resumo Esta revisão visa a conhecer e analisar os efeitos dos probióticos na depressão, ansiedade e estresse psicológico. Esses distúrbios estão entre as principais causas de incapacidades no mundo. As farmacoterapias convencionais costumam apresentar fraca resposta ou efeitos colaterais adversos. Estudos mais recentes têm demonstrado uma densa comunicação bidirecional chamada de eixo cérebro-intestino. Pesquisas estão evidenciando as relações entre alterações no microbioma entérico e distúrbios psiquiátricos, abrindo caminho para o surgimento de terapêuticas alternativas. Fez-se uma busca sistemática por ensaios clínicos randomizados duplo/triplo cego controlados por placebo no PubMed, Scopus e Lilacs. Para seleção dos estudos usaram-se as recomendações dos principais itens para relatar revisões sistemáticas e meta-análises (PRISMA). Nove artigos preencheram os critérios e foram analisados quanto aos efeitos na depressão, ansiedade, estresse psicológico e em biomarcadores. Sete encontraram resultados positivos em ao menos um dos itens. Conclui-se que o uso de probióticos para aliviar sintomas desses distúrbios é promissor, principalmente, por seu potencial efeito anti-inflamatório, mas são necessários ensaios clínicos randomizados duplo-cegos mais amplos e rigorosos para balizar tais conclusões.

Palavras-chave Depressão, Ansiedade, Probióticos, Microbiota, Eixo cérebro-intestino 


\section{Introdução}

A depressão e a ansiedade são distúrbios psiquiátricos heterogêneos complexos ${ }^{1} e$ umas das principais causas de incapacidades no mundo. Estima-se que cerca de $4,4 \%$ da população mundial, isto é, cerca de 320 milhões de pessoas, sofram de depressão e que o transtorno de ansiedade afete mais de 260 milhões $^{2}$. Segundo um estudo ${ }^{2}$ realizado pela Organização Mundial da Saúde (OMS), o Brasil é líder mundial na prevalência de transtornos de ansiedade (9,3\% da população) e ocupa o quinto lugar nas taxas de depressão (cerca de 5,8\% dos brasileiros, o que corresponde a 11,5 milhões de casos).

Em países de baixa e média renda, principalmente, a prevalência desses transtornos mentais está aumentando, inclusive com pessoas experimentando transtornos de depressão e ansiedade simultaneamente ${ }^{2,3}$. Os sintomas da depressão, tais como perda de interesse pela vida, desesperança, alterações no sono e no apetite, podem ser duradouros ou recorrentes e impactam negativamente as funções da vida diária dos indivíduos. A ansiedade excessiva/suprafisiológica com mudanças intensas no comportamento, preocupações generalizadas, desconforto gastrointestinal, respiratório e tensões musculares ${ }^{4}$ também afeta profundamente a rotina dos indivíduos, sua performance escolar ou laboral e sua qualidade de vida $^{5}$. Ambos os transtornos estão também associados ao aumento de risco para doenças cardíacas coronarianas, abuso de álcool e outas substâncias danosas ${ }^{6}$.

Em 1991, Smith ${ }^{7}$ propôs a teoria da depressão dos macrófagos, na qual afirmou que desequilíbrios imunológicos e inflamatórios são os principais fatores que levam ao surgimento e/ ou manutenção da depressão. Segundo o autor, o intestino exerceria o papel de ativação imune. Em conformidade com essa teoria, o eixo cérebro-intestino possui uma comunicação bidirecional entre o sistema nervoso central (SNC), o entérico (SNE) e o sistema endócrino, conectando os centros emocionais e cognitivos do cérebro às funções intestinais periféricas ${ }^{8,9}$. $\mathrm{O}$ avanço do conhecimento científico na direção que congrega áreas como psiquiatria, nutrição, gastrenterologia e neurologia, entre outras, tem estabelecido como certa, a extensa comunicação bioquímica existente entre o sistema nervoso central e o trato gastrointestinal $(\mathrm{TGI})^{10}$.

O TGI humano é habitado por quase 100 trilhões de microrganismos, conhecidos coletivamente como microbiota intestinal ${ }^{11}$. A interação desses microrganismos com o metabolismo humano é tão profunda que, segundo Gill et al. ${ }^{11}$, os seres humanos são superorganismos cujo metabolismo representa uma amálgama de atributos microbianos e humanos. Existem evidências que a microbiota intestinal interage intimamente com o principal sistema neuroendócrino: o eixo hipotálamo-hipófise (pituitária)-adrenal (HPA), que controla vários processos corporais em resposta ao estresse. A comunicação entre a microbiota intestinal e o eixo HPA também está profundamente relacionada a outros sistemas, como mencionado anteriormente ${ }^{12}$. Desse modo, da mesma forma que uma resposta fisiológica apropriada e coordenada - como uma resposta imune ao estresse - é necessária para a sobrevivência, uma resposta disfuncional pode ser prejudicial para o hospedeiro, contribuindo para o desenvolvimento de vários distúrbios do $\mathrm{SNC}^{13}$.

Logan e Katzman ${ }^{14}$ foram os primeiros a sugerir a modulação da microbiota intestinal com o uso de probióticos como adjuvante no tratamento da depressão. Depois deles, um crescente número de estudos clínicos e pré-clínicos vêm corroborando essa teoria. Ensaios em animais germ-free, por exemplo, mostraram que a colonização bacteriana do intestino é fundamental para o amadurecimento e desenvolvimento do sistema nervoso entérico e central ${ }^{8}$. A ausência desta colonização está associada a alterações na expressão de neurotransmissores em ambos os sistemas e a diversas disfunções motoras e sensoriais do TGI. Após a colonização dos animais com espécies específicas bacterianas, as anomalias são restauradas 8 .

Do mesmo modo, estudos científicos têm evidenciado que a microbiota intestinal desempenha funções essenciais que impactam na integridade da parede intestinal, na secreção de citocinas, na supressão da inflamação intestinal e na restauração da estrutura de suas junções apertadas (TJ, sigla em inglês) ${ }^{15}$. Portanto, em consonância com a teoria dos macrófagos e o estudo de Logan e Katzman ${ }^{14}$, a eubiose do microbioma entérico pode ter um efeito benéfico na inflamação e na adequada comunicação do eixo cérebro -intestino. Tais achados estão em harmonia com as observações de Dean e Keshavan ${ }^{1}$. Esses autores afirmam que, embora a exata fisiopatologia da depressão continue desconhecida, há padrões inflamatórios com hiperatividade do eixo HPA e com redução da neuroplasticidade que acarretam disfunções nas neurotransmissões ${ }^{1}$. O estresse psicológico, destarte, demonstra aumentar a produção de citocinas comointerleucina (IL) 
$-1 \beta,-6$ e fator de necrose tumoral (TNF)- $\alpha$ evidenciando um feedback positivo entre depressão e inflamação. Nesse processo, a inflamação causa depressão e estresse psicológico, que por sua vez é pró-inflamatório ${ }^{1,16}$.

Embora existam vários tratamentos farmacológicos bem estabelecidos para a ansiedade e a depressão, muitos pacientes experimentam uma fraca resposta ou efeitos colaterais adversos, como náusea, agitação, dores de cabeça, sonolência e disfunção sexual ${ }^{5}$. Diante desse contexto, o surgimento de terapêuticas alternativas como a administração de determinados probióticos para manipular o microbioma entérico com intuito de melhorar sintomas psicológicos de ansiedade e de depressão, pode ser um campo muito promissor. Dinan ${ }^{17}$ e colaboradores, denominaram de psicobióticos aqueles microrganismos vivos que, quando ingeridos em quantidades adequadas, produzem benefícios para a saúde mental.

A presente revisão sistemática tem como objetivo elucidar os efeitos do uso de probióticos na depressão, na ansiedade e no estresse psicológico em seres humanos saudáveis - podendo apresentar um ou vários destes sintomas psicológicos. Nesse intuito, serão analisados os mais recentes ensaios clínicos randomizados controlados por placebo encontrados nas bases de dados selecionadas.

\section{Métodos}

\section{Estratégia de busca}

Foi realizada uma busca sistemática e informatizada, de acordo com as diretrizes PRISMA ${ }^{18}$, nas bases de dado Pubmed, Scopus e Lilacs. As listas de referência dos artigos identificados foram cuidadosamente pesquisadas para obter possíveis informações adicionais. A estratégia utilizada consistiu na recuperação de artigos através do uso dos termos "depressive disorder" ou "depression" ou "anxiety" e "probiotic", no período que abrange primeiro de janeiro de 2015 a 31 de março de 2020, com filtro aplicado para ensaios em humanos, nos idiomas inglês, espanhol e português. A busca foi realizada no dia primeiro de abril de 2020. Os resultados e as etapas deste processo são apresentados no fluxograma abaixo (Figura 1).

\section{Critérios de seleção do estudo}

A seleção incluiu estudos duplo e triplo-cegos, randomizados e controlados por placebo re- alizados em humanos (com idade média maior que 16 e até 65 anos) que analisaram os efeitos do consumo de suplemento probiótico nos sintomas de depressão, ansiedade, níveis de cognição, estresse ou estado de humor. Não houve restrição quanto à dosagem, cepas ou forma de administração dos probióticos. Os critérios de exclusão incluíram: estudos em animais; em crianças (idade média $\leq 16$ anos) ou em adultos mais velhos (idade média $>65$ anos); em gestantes; em adultos não saudáveis (exceto nos que apresentavam depressão, ansiedade ou estresse), estudos longitudinais e ausência de escala para mensuração de ansiedade e depressão. Capítulos de livro, cartas ao editor e resenhas foram excluídos. Igualmente, foram retirados da análise qualitativa ensaios clínicos que utilizaram outras substâncias - farmacológicas ou não - em conjunto com cepas probióticas.

\section{Desfecho}

O desfecho primário esperado é o esclarecimento dos efeitos psicológicos da administração de probióticos na depressão e na ansiedade ou no estresse psicológico, utilizando-se pelo menos uma escala psicológica para avaliação dos sintomas. Secundariamente, neste trabalho, são examinados os processos bioquímicos que podem fazer parte da fisiopatologia da depressão.

\section{Extração de dados e risco de viés}

Os seguintes dados foram extraídos de cada estudo: autores e ano; características da amostra; número de indivíduos; desenho do estudo; duração da intervenção; cepas, número de bactérias viáveis (expresso em UFC, unidades formadoras de colônias) e dose; modalidade de administração dos probióticos; sintomas psicológicos e escalas de mensuração; resultados e principais achados. O título e o resumo de cada resultado da pesquisa foram triados por duas revisoras que aplicaram independentemente os mesmos critérios de inclusão e exclusão. Não houve dissenso.

\section{Resultados}

Como mostrado na Figura 1, inicialmente foram encontrados 119 artigos. Após a primeira triagem para excluir as duplicidades, chegou-se a 107 artigos. Destes, 16 foram submetidos a análise do texto completo e apenas nove artigos atenderam aos critérios de seleção estabelecidos na metodologia. 


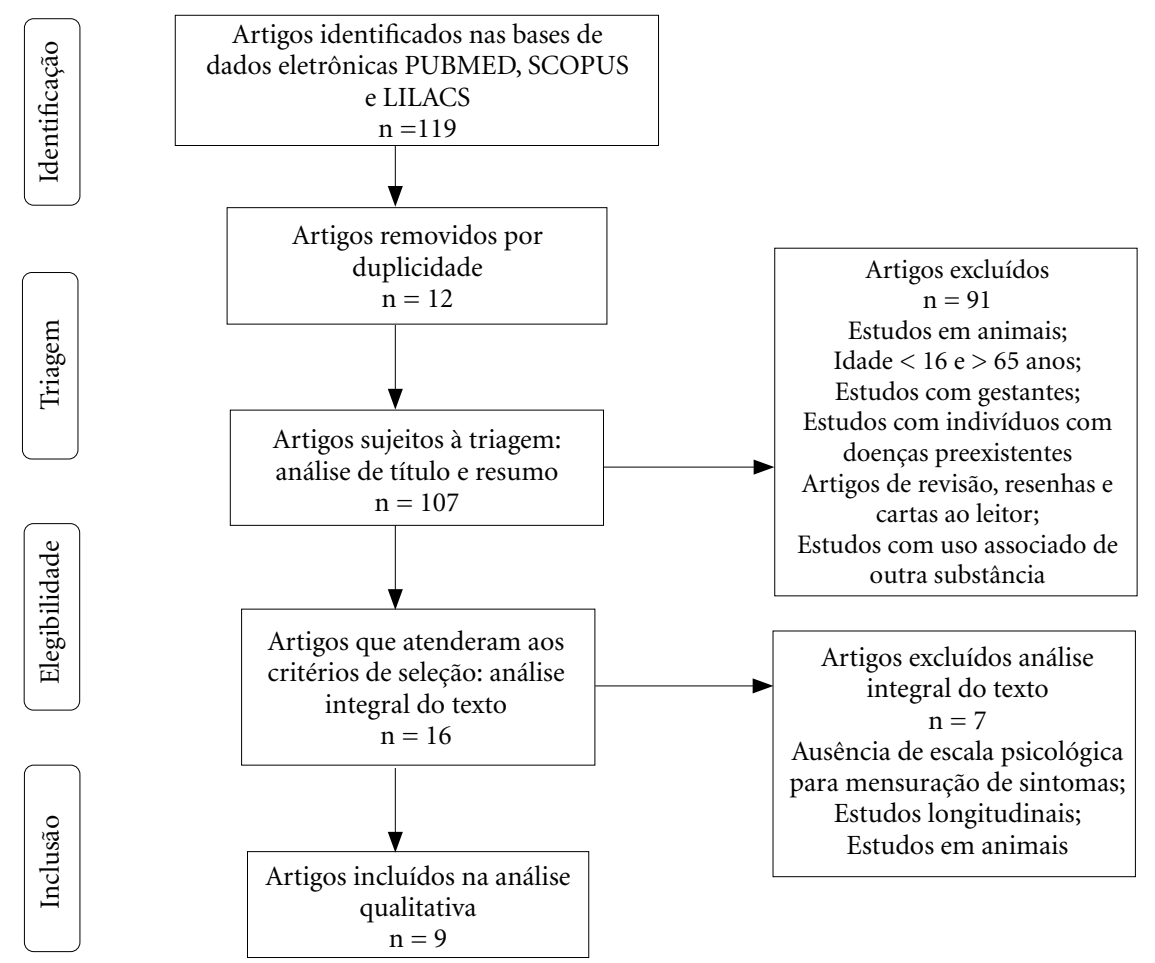

Figura 1. Fluxograma do processo sistemático de pesquisa e seleção de literatura conforme a recomendação PRISMA.

Fonte: Elaborado pelas autoras.

Esta revisão, portanto, inclui a análise de nove estudos $^{19-27}$ cujas características estão descritas na Tabela 1. Oito dos selecionados ${ }^{19-26}$ tratam de ensaios clínicos duplo-cegos randomizados, controlados por placebo. Nesse conjunto, um é um estudo cruzado ${ }^{22}$ e outro foi realizado três vezes durante três anos consecutivos ${ }^{26}$. Apenas um dos trabalhos consiste num ensaio randomizado triplo-cego ${ }^{27}$, controlado por placebo pré e pós intervenção. $\mathrm{O}$ tempo de intervenção dos estudos variou entre quatro e 12 semanas e o total de indivíduos randomizados foi de 698 .

Dois estudos ${ }^{19,21}$ avaliaram pacientes estressados, um estudo ${ }^{23}$ avaliou adultos com alteração moderada de humor e, outro, adultos com depressão $^{24}$. Os demais trabalhos ${ }^{20,22,25-27}$ avaliaram pacientes saudáveis sem sintomas de transtornos psicológicos ou psiquiátricos. Em um deles ${ }^{25}$, a amostra foi composta por trabalhadores petroquímicos; e outro apenas por indivíduos do sexo masculino ${ }^{22}$.

Todos os estudos selecionados utilizaram questionários e escalas para avaliar sintomas de depressão e/ou ansiedade nos pacientes. Todos os ensaios clínicos consistiram na administração de espécies bacterianas comensais, podendo ser com o uso de apenas uma cepa ${ }^{19,21,22,26}$ ou mistura de cepas probióticas. São os seguintes os gêneros dos microrganismos administrados:Lactobacilos (L.), Bifidobacterias (B.), Streptococcus(S.) e/ou Lactococcus. Os mais utilizados foram os Lactobacilos e não houve uma cepa mais empregada.Dois estudos $^{24,25}$ não especificam as cepas das espécies bacterianas usadas. ETran et al. ${ }^{20}$ não citam quais microrganismos utilizaram, mencionam apenas o número de cepas administrado para cada grupo amostral.

\section{Efeitos na depressão}

Seis estudos ${ }^{19,21,23-25,27}$ avaliam os efeitos da administração diária de probiótico na depressão, com intervenções que variaram entre quatro e 12 semanas. Apenas um deles examinou somente os sintomas de depressão $0^{24}$. Todos os demais analisaram também outras alterações psicológicas, tais como ansiedade e estresse psicológico. Para qualificar e quantificar as alterações nos sintomas psicológicos os autores utilizaram questionários com escalas validadas cientificamente. 


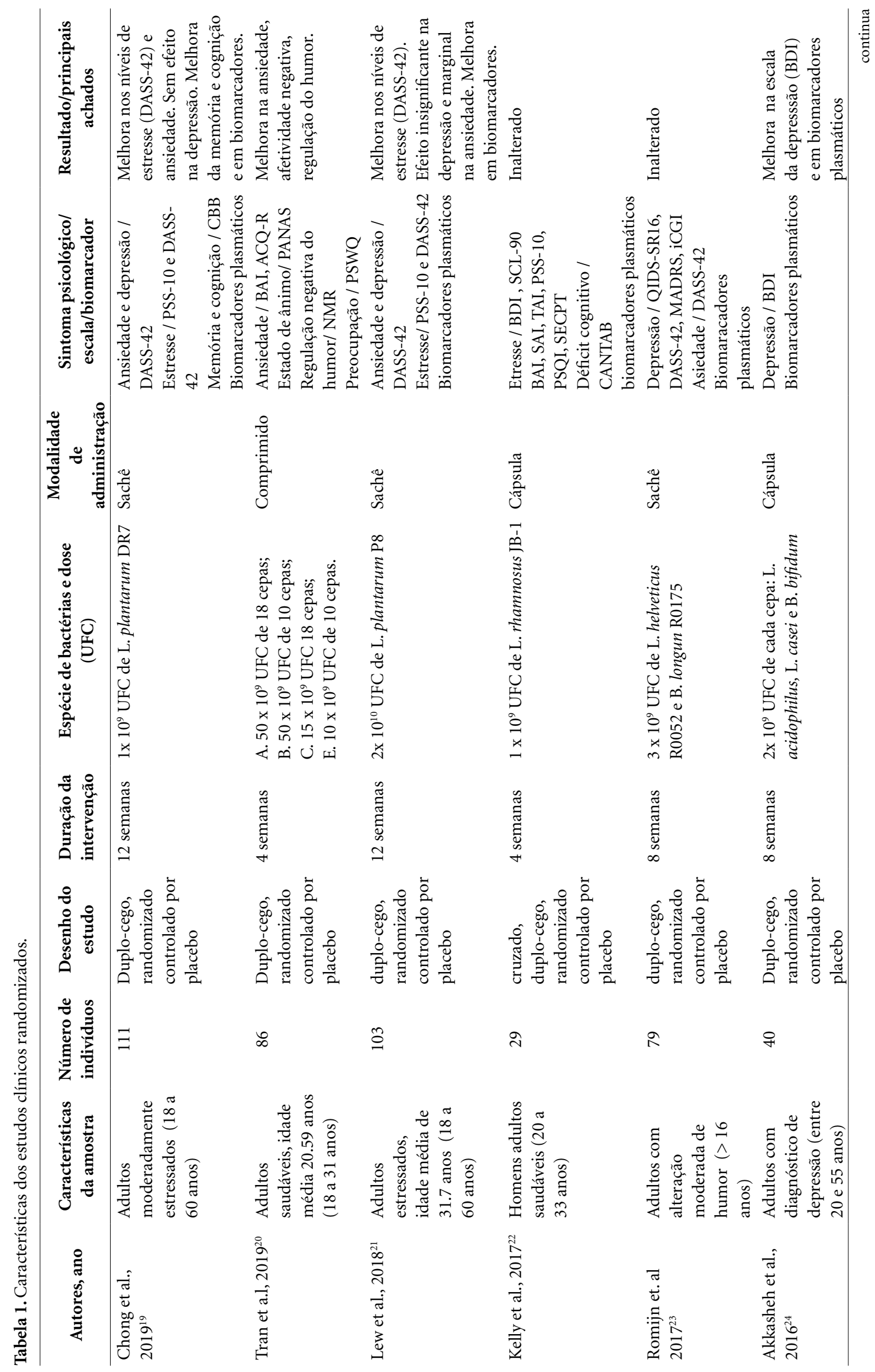




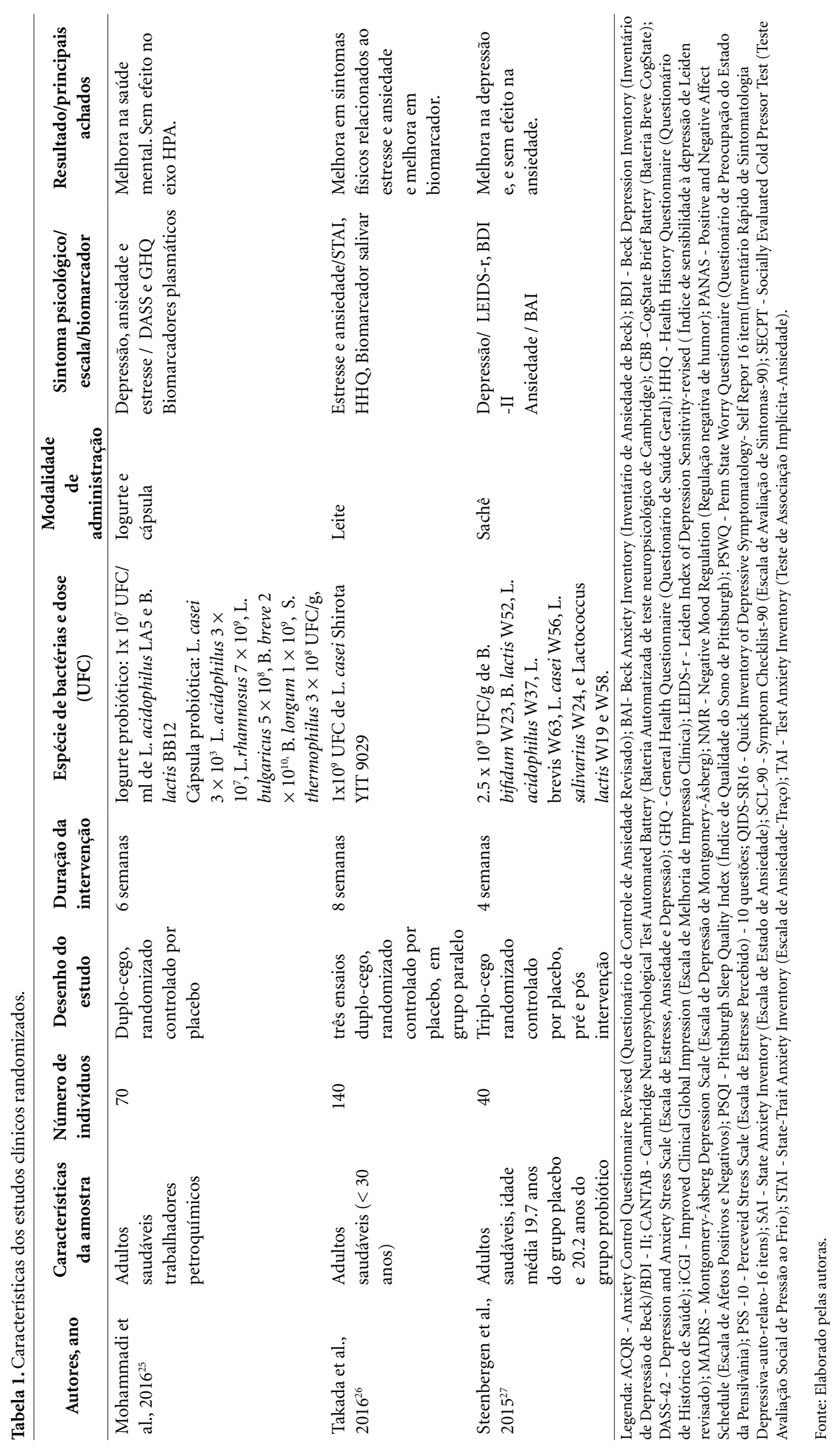


Três ${ }^{24,25,27}$ dos seis estudos relataram melhora nos sintomas depressivos e os outrostrês ${ }^{19,21,23}$ concluem que não houve alteração nos sintomas ou esta foi insignificante. No entanto, entre os trabalhos que não encontraram alterações significativas, Lew $^{21}$ e colaboradores concluíram que o L. plantarum $\mathrm{P} 8$ tem efeitos positivos em outros sintomas psicológicos - que serão abordados mais adiante - avaliados pela DASS-42. Igualmente, Chong et al. ${ }^{19}$, encontraram que o L. plantarumDR7, apesar de não demonstrar efeitos benéficos nos itens relativos à depressão na DASS-42, sua administração resulta em sintomas reduzidos de estresse e ansiedade, e em melhora de várias funções cognitivas e de memória, em níveis reduzidos de cortisol plasmático e de citocinas pró-inflamatórias. Romijn et al. ${ }^{23}$, por sua vez, ainda que não tenham encontrado diferença significativa em qualquer medida de resultado psicológico entre os grupos que ingeriram o sachê contendo L. helveticusR0052 e B. longum R0175 e aqueles com placebo, concluíram que o nível basal de vitamina $\mathrm{D}$ moderou o efeito positivo do tratamento, que é verificado em várias escalas utilizadas.

Com relação aos resultados positivos para a melhora dos sintomas da depressão, Akkasheh ${ }^{24}$ e seus colegas observaram que após oito semanas de intervenção, pacientes deprimidos que receberam suplementos probióticos - contendo L. casei, L acidophilus e B. bifidum - diminuíram significativamente a pontuação total do BDI em comparação com placebo, indicando melhora geral dos sintomas, incluindo do humor. Adicionalmente, esses sujeitos apresentaram uma evolução significativa em alguns biomarcadores plasmáticos, que serão analisados mais adiante. Mohammadi et al..$^{25}$ analisaram os efeitos de duas intervenções probióticas: 1) Iogurte probiótico contendo L. acidophilus LA5 e B. lactis BB12 e 2) cápsula probiótica contendo L. casei, L. acidophilus, L rhamnosus, L. bulgaricus, B. breve, B. longum, S. thermophilus, em trabalhadores petroquímicos saudáveis. Após seis semanas de tratamento, foi observada uma melhora significativa da saúde mental avaliada pelo GHQ e pelos escores da DASS, o mesmo não tendo sido detectado no grupo que recebeu iogurte convencional. Os autores não apresentaram as pontuações separadas por sintoma psicológico. Por fim, Steenbergen et al. encontraram que, após quatro semanas de intervenção probiótica multi-espécies (B. $b i$ fidum W23, B. lactis W52, L. acidophilus W37, L. brevis W63, L. casei W56, L. salivarius W2 e Lactococcuslactis W19 e W58) em indivíduos saudá- veis, os participantes mostraram uma reatividade cognitiva significativamente reduzida à depressão - avaliadas pelo LEIDS-r, principalmente pela redução da ruminação e dos pensamentos agressivos.

\section{Efeitos na ansiedade e estresse psicológico}

Oito $^{19-23,25-27}$ dos nove estudos incluídos nesta revisão avaliaram estresse e/ou ansiedade e cinco relataram melhora nos sintomas após a intervenção com probiótico. Entre estes, $u^{26}$, ainda que não tenha encontrado evolução na escala psicológica, descreveu efeitos positivos nos sintomas físicos e em um biomarcador de estresse. Entre os três que não observaram impacto positivo, Kelly ${ }^{22}$ e seus colegas, em seu ensaio com 29 adultos homens saudáveis, não encontraram efeito benéfico do uso de L. rhamnosus JB-1 nas medidas de ansiedade, estresse, humor ou qualidade do sono. Tampouco, nas medidas subjetivas de estresse ou na resposta do eixo HPA ao SECPT. No mesmo sentido, Romjin et al. ${ }^{23}$ também não observaram nenhuma diferença significativa entre os grupos probiótico e placebo em qualquer medida de resultado psicológico após oito semanas. Steenbergen ${ }^{27}$ e seus colegas não encontraram efeitos significativos da intervenção nos sintomas ansiedade, mas atribuíram ao fato da escala de BAI tratar de um autorrelato de ansiedade e os indivíduos selecionados apresentarem pontuações mínimas ou leves no começo do tratamento.

No recente estudo levado a cabo por Chong et al. ${ }^{19}$, os autores concluíram que o consumo de DR7 reduziu os sintomas de estresse e ansiedade - avaliado pelo questionário DASS-42 - em até oito semanas em adultos estressados comparados ao grupo placebo. Esses autores dividiram os indivíduos estudados em dois grupos (idade abaixo e acima de 30 anos) tanto para aqueles que receberam o probiótico como para o placebo. $\mathrm{O}$ estresse foi avaliado por meio do questionário PSS-10 e do DASS-42. Segundo essa pesquisa, a administração de DR7 apresenta maior benefício para os adultos jovens ( $<30$ anos), com maior redução nos escores totais de estresse da DASS42 após a oitava semana em comparação com o grupo que recebeu placebo. A administração de DR7 também reduziu as pontuações de ansiedade em todas as populações estudadas - avaliada pela mesma escala. Ademais, o DR7 melhorou as funções cognitivas e de memória em adultos maiores de 30 anos. Ou seja, de acordo com autores, embora o probiótico tenha beneficiado tanto 
a população jovem quanto os mais velhos em relação aos sintomas de ansiedade, seu uso favorece unicamente à população adulta jovem na avaliação do estresse.

Tran et al. ${ }^{20}$, também em um ensaio clínico realizado recentemente, dividiram sua amostra de adultos saudáveis em cinco grupos. O grupo A recebeu comprimidos de probiótico com número alto de UFC e de espécies de microrganismos; o B recebeu um alto número de UFC e baixo de espécies; o C foi o grupo controle, que ingeriu placebo; o D recebeu um número baixo de UFC e alto de espécies e, por fim, o E recebeu um número baixo de ambos (veja tabela 1). Os achados do estudo sugerem que os probióticos melhoraram o pânico, a ansiedade neurofisiológica, o afeto negativo, a preocupação e a regulação negativa do humor. Adicionalmente, conclui-se que a quantidade de UFC é mais eficaz do que o número de espécies, ao contabilizar o número de melhorias significativas. Por fim, observam que os participantes que relataram maior sofrimento foram também os que apresentaram melhor evolução.

Lew et al. ${ }^{21}$ observaram que, após 12 semanas, indivíduos estressados que consumiram L. plantarum P8 apresentaram pontuações reduzidas de estresse e ansiedade comparados ao placebo após a quarta semana - avaliado pela DASS-42. A eficácia do P8 na redução do estresse, em comparação com o placebo, foi atribuída predominantemente à redução da irritabilidade, irritação, aumento da calma e da tolerância. Quanto à ansiedade, o P8 mostrou-se eficiente, principalmente, na redução da falta de ar, nos batimentos cardíacos anormais não relacionados às atividades físicas e na diminuição do medo do desconhecido. Secundariamente, a intervenção teve efeitos positivos na velocidade da cognição social e emocional nas mulheres, enquanto os homens não exibiram diferenças em comparação ao placebo.

Por três anos consecutivos, Takada ${ }^{26}$ e sua equipe analisaram os efeitos do L.casei Shirota nas respostas psicológicas e fisiológicas em estudantes de medicina saudáveis. Os autores concluíram que subiu o nível de estresse psicológico - segundo o STAI - antes de realizarem um exame nacional. Com a proximidade do exame, tanto o grupo placebo como o probiótico tiveram pontuações aumentadas para o estresse. No entanto, aqueles que tomaram o L.c Shirota YIT 9029 reduziram significativamente o aumento do cortisol salivar e apresentaram diminuição expressiva dos sintomas físicos, incluindo queixas abdominais. A supressão induzida pelo L.c Shirota da secreção salivar de cortisol pode ter sido associada ao alívio dos sintomas físicos associados ao estresse ${ }^{26}$.

Finalmente, como já descrito na seção anterior, Mohammadi ${ }^{25}$ e seus colegas avaliaram os efeitos do uso de probióticos para ansiedade, estresse e depressão segundo a DASS-42 e o GHQ e comprovaram melhora significativa da pontuação global.

\section{Efeitos em biomarcadores inflamatórios, hormonais e neuro-hormonais}

Além da avaliação dos sintomas psicológicos, seis $^{19,21-25}$ ensaios incorporaram o exame de biomarcadores plasmáticos e $\mathrm{um}^{26}$ de cortisol salivar. Dentre esses sete estudos, três ${ }^{22,23,25}$ não encontraram impacto da intervenção probiótica nos marcadores avaliados.

Chong $^{19}$ e seus colaboradores analisaram as amostras de plasma quanto às concentrações de cortisol, IL-1 $\beta,-4$ e -10, TNF- $\alpha$ e interferon (IFN) $-\gamma$. Os pesquisadores encontraram que o nível de cortisol plasmático foi reduzido entre os indivíduos que receberam o DR7 em comparação com o placebo, acompanhado da diminuição de citocinas pró-inflamatórias - IFN- $\gamma$ e TNF- $\alpha$ - e do aumento das anti-inflamatórias, como a IL-10. Quando os resultados foram analisados por grupos etários, verificaram que o DR7 exerceu efeitos mais prevalentes no aumento das citocinas anti-inflamatórias IL-10 e na diminuição das pró -inflamatórias IFN- $\gamma$ em adultos jovens, enquanto reduziu apenas as citocinas pró-inflamatórias IFN- $\gamma$, TNF- $\alpha$ e IL- $1 \beta$ em adultos mais velhos, sem qualquer efeito sobre as anti-inflamatórias. Do ponto de vista da sinalização neuro-hormonal, a administração de DR7 melhorou a via da serotonina, por expressões reduzidas de dopamina $\beta$-hidroxilase $(\mathrm{DBH})$, tirosina hidroxilase (TH), indoleamina 2,3-dioxigenase (IDO) e triptofano 2,3-dioxigenase (TDO), acompanhadas por valores aumentados de triptofanohidroxilase (TPH)-2 e 5-hidroxitriptamina (HT)-6. Paralelamente, a via da dopamina foi regulada com a estabilização dos níveis de TH e DBH.Chong et al. ${ }^{19}$, portanto, observaram que o tratamento probiótico pode exercer efeitos benéficos através de mecanismos que envolvem a regulação positiva das vias da serotonina e a estabilização das vias da dopamina ao longo do eixo cérebro-intestino. Por fim, o uso de DR7 não afetou as expressões de descarboxilase do ácido glutâmico (GAD)-65, ácido gama-aminobutírico $\alpha-5$ (GABA5), fator neurotrófico derivado do cérebro (BDNF) e elementos de resposta a cAMP (CRE). 
$\mathrm{Lew}^{21}$ e seus colegas não encontraram diferença significativa nos níveis plasmáticos de cortisol, mas observaram uma redução importante do IFN- $\gamma$ nos sujeitos que receberam o probiótico, enquanto houve aumento no grupo placebo. Os valores de TNF- $\alpha$ aumentaram nos dois grupos. Mas, aqueles administrados com P8, apresentaram um aumento menor em comparação com os que ingeriram placebo, principalmente no caso das mulheres. Ambos os grupos não produziram alterações nos níveis plasmáticos de IL-10, IL-1 $\beta$ e IL-4 durante o estudo.

Romijn et al. ${ }^{23}$ observaram que $75 \%$ de sua amostra tinham pelo menos um marcador de inflamação analisado (proteína C-reativa - PCR, IL-1 $\beta$, IL- 6 ou TNF- $\alpha$ ) elevado. Após o tratamento, os pesquisadores não encontraram diferenças significativas nos níveis dos biomarcadores entre os que consumiram o probiótico e o grupo placebo. No entanto, entre aqueles randomizados para o grupo probiótico, os indivíduos com valores mais altos de vitamina $\mathrm{D}$ no início do estudo apresentaram efeitos mais positivos no humor do que os que possuíam níveis mais baixos. Indicando, segundo os autores, um papel relevante da vitamina D na moderação dos efeitos da intervenção.

Kelly et al. ${ }^{22}$ não encontraram alterações com o uso de L. rhamnosus JB-1 nas concentrações de IL-10, IL-1 $\beta$, IL-6 ou IL-8. Deste modo, não foram observados efeitos anti-inflamatórios significativos, avaliados pelos níveis de citocinas basais e estimuladas.

$\mathrm{Na}$ avaliação das amostras plasmáticas realizadas por Akkasheh ${ }^{24}$ e seus colaboradores, foram observados decréscimos expressivos nos níveis séricos de insulina, HOMA-IR (modelo de avaliação da homeostase da resistência à insulina) e concentrações menores de PCR após a suplementação com probióticos, comparados com o placebo. Ademais, a ingestão de probióticos resultou em aumento significativo dos valores plasmáticos de glutationa (GSH) em relação ao placebo. Porém, os autores não encontram mudanças relevantes nos níveis de glicemia de jejum, HOMA- $\beta$ (células $\beta$ pancreáticas), QUICKI (índice quantitativo de sensibilidade à insulina), perfis lipídicos e capacidade antioxidante total (CAT) após a suplementação.

Mohammadi et al. ${ }^{25}$ revelaram que a suplementação com probióticos em trabalhadores petroquímicos por seis semanas não afetou biomarcadores do eixo HPA, tais como quinurenina, triptofano, neuropeptídeo Y e cortisol via hormônio adrenocorticotrófico (ACTH).
Por fim, Takada ${ }^{26}$ e seus colaboradores testaram amostra de cortisol salivar e concluíram que o seu aumento induzido pelo estresse foi significativamente suprimido no grupo que consumiu o leite com probiótico em comparação com o grupo que ingeriu placebo.

\section{Discussão}

Esta revisão procurou entender os efeitos do uso de probióticos em algumas desordens mentais, especialmente na depressão, ansiedade e no estresse psicológico. Quando analisados em conjunto, os achados dos ensaios clínicos que avaliaram os efeitos sobre a depressão não permitem chegar a uma conclusão definitiva a respeito da eficácia de uma ou de várias cepas probióticas. Três estudos ${ }^{19,21,23}$ com um total de 293 sujeitos randomizados, demonstraram que o consumo de probióticos não trouxe benefícios para os sintomas de depressão. Outros três, realizados com um total de 150 adultos, observaram efeitos benéficos. No entanto, Mohammadi et al. ${ }^{25}$ não especificaram quais parâmetros da escala DASS impactaram significativamente no tratamento com microrganismos, limitando a possibilidade de se conhecer o real alcance da intervenção levada a cabo.

Os indicadores psicológicos de ansiedade e estresse, analisados conjuntamente, apresentaram melhor evolução quando comparados aos de depressão. É possível que mediante as condições psicológicas mais profundas e severas presentes na depressão (que incluem estados mentais deteriorados, como disforia, desesperança, desvalorização da vida e, autodepreciação), sejam necessários estudos que proponham estratégias de intervenções mais prolongadas ou mesmo de forma adjuvante a tratamentos farmacológicos. Apesar dos avanços nas pesquisas da doença, a depressão continua sendo um distúrbio desafiador para o tratamento. Isso se deve, entre outras razões, aos numerosos efeitos colaterais dos fármacos hoje disponíveis e por estes, geralmente, induzirem a remissão do transtorno depressivo maior em cerca de um terço dos pacientes apenas $^{28}$. Portanto, identificar novas terapêuticas é fundamental.

Observa-se, todavia, que a variabilidade no uso de cepas probióticas, na dosagem, na duração da intervenção e da própria característica da amostra evidenciam limitações importantes para alcance de um consenso quanto os efeitos benéficos do seu uso para os distúrbios analisados. Ade- 
mais, os estudos não examinaram preliminarmente as condições de eubiose/disbiose entérica dos indivíduos randomizados, que podem exercer forte impacto nos resultados encontrados.

Do ponto de vista dos biomarcadores, estu$\operatorname{dos}^{29}$ demonstram que a depressão está associada com ativação da resposta inflamatória, com aumento de proteínas de fase aguda positiva, incluindo a PCR, e de citocinas pró-inflamatórias como a IL-1, IL-2, IL-6 e IFN- . Padrões séricos semelhantes de marcadores foram observados em estados de ansiedade e são conhecidos por ocorrer como resultado do estresse ${ }^{17}$.

No presente estudo, quatro ${ }^{19,21,24,26}$ ensaios observaram alterações em biomarcadores após a intervenção com probióticos. Os trabalhos de Chong et al. ${ }^{19} \mathrm{e} \mathrm{de} \mathrm{Lew} \mathrm{et} \mathrm{al.} .^{21}$ foram os únicos que examinaram o impacto nos índices de TNF- $\alpha$, IFN- $\gamma$, IL-1 $\beta$, -4 e -10. Ambos os grupos encontraram redução significativa do IFN- $\gamma$ e TNF- $\alpha$, ainda que $\mathrm{Lew}^{21}$ e sua equipe tenham constatado diminuição relativa do último marcador. Quanto às demais citocinas, Chong et al. ${ }^{19}$ concluíram que o DR7 reduziu expressivamente seus níveis. Já $L^{2} w^{21}$ e colaboradores não viram efeito do $\mathrm{P} 8$ nesses biomarcadores. Akkasheh et al. ${ }^{24}$ observaram uma diminuição significativa nos níveis de insulina, HOMA-IR, de PCR e aumento de GSH. Enquanto, Takada et al..$^{26}$ observaram a supressão do aumento dos níveis de cortisol salivar.

Esses resultados são indícios importantes de que determinados microrganismos podem ter impacto positivo no perfil inflamatório de adultos. Como comentado anteriormente, há evidências ${ }^{19,21,30}$ de que pacientes deprimidos, estressados ou ansiosos apresentam um perfil aumentado de citocinas pró-inflamatórias e proteínas de fase aguda positiva. Ademais, Dinan ${ }^{17} \mathrm{e}$ colaboradores observaram que estudos com roedores indicam que o estresse altera a função da barreira intestinal, permitindo que lipopolissacarídeos (LPS) e outras moléculas tenham acesso à corrente sanguínea, estimulando o receptor do tipo Toll 4 (TLR4) e outros TLRs, resultando na produção de citocinas inflamatórias. Portanto, são intrínsecas as relações entre o intestino, o perfil inflamatório/imunológico e as respostas neuroendócrinas.

$\mathrm{Na}$ literatura analisada, parece haver duas hipóteses dominantes abordando possíveis mecanismos de ação através dos quais os probióticos exercem seus efeitos na saúde mental. Essas teorias envolvem a regulação de marcadores inflamatórios e a neurotransmissão da serotonina. No entanto, como o sistema imunológico, o siste- ma nervoso central e o sistema nervoso entérico estão tão intricadamente conectados, talvez seja possível afirmar que os dois mecanismos de ação trabalhem em conjunto para produzir os efeitos induzidos pelos probióticos ${ }^{5}$. Em consonância com essa constatação, O'Brien et al. ${ }^{29}$ afirmam que distúrbios na função do eixo HPA há muito são reconhecidos como uma característica da depressão maior, especialmente em pacientes com características melancólicas. De acordo com os autores, está em desenvolvimento uma variedade de novos antidepressivos que têm como alvo os receptores nesse eixo e parece apropriado explorar locais imunes. Antagonistas de citocinas pró -inflamatórias como a IL-1 ou IL-6 reduziriam a unidade HPA e, ao fazê-lo, poderiam aliviar os sintomas depressivos. Uma estratégia alternativa seria a busca de novos compostos que aumentem a produção de citocinas anti-inflamatórias. Como resultado da presente revisão, o DR7 apresentou efeitos nessa direção. Ainda que não foram suficientes para impactar nos escores da DASS-42.

Segundo Dinan ${ }^{17}$ e colaboradores, os psicobióticos, como uma classe de probiótico, são capazes de produzir e distribuir substâncias neuroativas, como o GABA e a serotonina, que atuam no eixo cérebro-intestino. Muitas pesquisas sobre os psicobióticos são baseadas em modelos de roedores, que usam induções de estresse e testes comportamentais para avaliar motivação, ansiedade e depressão ${ }^{31}$.Os efeitos psicofisiológicos dos psicobióticos, segundo Sakar et al., se enquadram nas três categorias a seguir: (1) efeitos psicológicos nos processos emocionais e cognitivos; (2) efeitos sistêmicos no eixo HPA, na resposta ao estresse (glicocorticoide) e na inflamação caracterizada por concentrações enormes de citocinas pró-inflamatórias e (3) efeitos neurais em neurotransmissores e proteínas tais como GABA, glutamato e BDNF ${ }^{31}$. Em conformidade com essas categorias, a presente revisão encontrou que o DR7 ${ }^{19}$, o $\mathrm{P}^{21}$ e a mistura de L. casei, L. acidophilus e B. bifidum ${ }^{24}$ atuaram positivamente em ao menos duas dessas vias de ação. $\mathrm{O}$ estudo de Chong et al. ${ }^{19}$ demonstrou que o DR7 incidiu nas três vias propostas por Sakar et al. ${ }^{31}$. Lew et al. ${ }^{21}$ e Akkasheh et al. ${ }^{24}$ comprovaram benefícios no perfil inflamatório e nos processos emocionais.

Quanto aos efeitos neurais, o papel da microbiota intestinal na regulação da biossíntese de serotonina a partir de células intestinais enterocromafins foi recentemente estabelecido na literatura ${ }^{32}$. Sabe-se que aproximadamente $90 \%$ da serotonina é derivada destas células e 5\% dos 
neurônios do $\mathrm{SNE}^{33}$ e que as vias cerebrais da serotonina estão envolvidas na regulação da cognição e do humor ${ }^{34,35}$. Portanto, disfuncionalidades nestas vias podem contribuir para a comorbidades dos distúrbios gastrointestinais e do humor. Paralelamente, são conhecidos os benefícios do uso de probióticos para a integridade intestinal e para a microbiota saudável ${ }^{36-38}$ - fundamentais para a biossíntese da serotonina. Nesse sentido, ainda que os trabalhos incluídos nesta revisão não tragam conclusões definitivas para a pergunta que deu origem a este estudo, é animador que sete (dos nove) estudos tenham observado algum impacto positivo do uso de probióticos.

No entanto, embora as evidências mencionadas acima e os resultados de alguns ensaios incluídos nesta revisão exponham um terreno muito promissor e pujante para o avanço das pesquisas sobre depressão e outros distúrbios como ansiedade e estresse psicológico, estudos clínicos para embasar novas terapêuticas com o uso de probióticos são fundamentais. Sem dúvida, a grande diversidade de espécies microbianas que habita o trato gastrointestinal e a competência de algumas espécies para sintetizar moléculas neuroativas, fazem do trato digestivo um local com grandes possibilidades farmacocinéticas e com potencial capacidade de impactar a saúde mental.

\section{Conclusão}

Esta revisão sistemática revelou uma pesquisa preliminar limitada, porém promissora quanto aos efeitos dos probióticos nos sintomas de depressão e de ansiedade em humanos. Igualmente expôs lacunas e inconsistências nos estudos publicados até o momento. Algumas limitações observadas nos ensaios analisados no presente estudo impediram que fosse alcançado um consenso sobre quais são os efeitos benéficos dos probióticos para cada um dos distúrbios analisados. Para superar os principais limitantes, mais ensaios clínicos randomizados duplo-cegos controlados por placebo são necessárias para determinar a eficácia no alívio dos sintomas, bem como a duração ideal do tratamento, dosagem e cepa probiótica para alcançar efeitos positivos na depressão e na ansiedade.

Ainda assim, conclui-se que as evidências apresentadas nesta revisão demonstram que o uso de probióticos impactam no perfil inflamatório dos pacientes e na regulação da via da serotonina. Estes achados coadunam com o potencial promissor uso de probióticos na depressão e na ansiedade, tendo em vista as alterações no eixo HPA e dos níveis séricos de biomarcadores inflamatórios presentes em pacientes deprimidos. Adicionalmente, quando analisados em conjunto, sete dos nove estudos comprovaram algum benefício da administração de probióticos em termos de saúde mental. Esta conclusão corrobora com uma meta-análise ${ }^{39}$ recente que, embora tenha demonstrado o promissor uso de probióticos no alívio dos sintomas depressivos, enfatizou a necessidade de mais evidências com amostras maiores e ensaios clínicos randomizados mais rigorosos.

\section{Colaboradores}

MS Minayo trabalhou na concepção, delineamento metodológico, pesquisa, análise dos dados, redação e revisão final do texto. I Miranda trabalhou na concepção e análise dos dados. RS Telhado trabalhou na revisão final do texto. 


\section{Referências}

1. Dean J, Keshavan M. The neurobiology of depression: An integrated view. Asian J Psychiatr 2017; 27:101111.

2. World Health Organization (WHO). Depression and other common mental disorders: global health estimates. Geneva: WHO; 2017.

3. Hirschfeld RM. The comorbidity of major depression and anxiety disorders: recognition and management in primary care. Prim Care Companion J Clin Psychiatry [periódico na Internet] $2001 \mathrm{dez}$. [cited $2020 \mathrm{Apr}$. 5]; 3(6):244-254. Available from: https://pubmed. ncbi.nlm.nih.gov/15014592/

4. Haug TT, Mykletun A, Dahl AA. Are anxiety and depression related to gastrointestinal symptoms in the general population? Scand J Gastroenterol [periódico na Internet] 2002 mar. [cited 2020 Apr. 3]; 37(3):294298. Available from: https://pubmed.ncbi.nlm.nih. gov/11916191/

5. Wallace CJK, Milev R. The effects of probiotics on depressive symptoms in humans: a systematic review [correção publicada em Ann Gen Psychiatry. 2017 mar; 16:18]. Ann Gen Psychiatry [periódico na Internet] 2017 fev. [cited 2020 Apr. 10]; 16:14. Available from: https:/www.ncbi.nlm.nih.gov/pmc/articlesPM C5319175/

6. Reis DJ, Ilardi SS, Punt SEW. The anxiolytic effect of probiotics: a systematic review and meta-analysis of the clinical and preclinical literature. PLoS One [periódico na Internet] 2018 jun. [cited 2020 Apr. 9]; 13(6). Available from: https://pubmed.ncbi.nlm.nih. gov/29924822/

7. Smith RS. The macrophage theory of depression. Med Hypotheses [periódico na Internet] 1991 ago [cited 2020 Mar 28]; 35(4):298-306. Available from: https://www.sciencedirect.com/science/article/abs/ pii/030698779190272Z

8. Carabotti M, Scirocco A, Maselli MA, Severi C. The gut-brain axis: interactions between enteric microbiota, central and enteric nervous systems. Ann Gastroenterol [periódico na Internet]. 2015 abril-jun. [cited 2020 Mar 22]; 28(2):203-209. Available from: https:// www.ncbi.nlm.nih.gov/pmc/articles/PMC4367209/

9. Foster JA, McVey Neufeld KA. Gut-brain axis: how the microbiome influences anxiety and depression. Trends Neurosci [periódico na Internet] 2013 maio [cited 2020 Apr. 9]; 36(5):305-312. Available from: https://pubmed.ncbi.nlm.nih.gov/23384445/

10. Grenham S, Clarke G, Cryan JF, Dinan TG. Brain-gutmicrobe communication in health and disease. Front Physiol [periódico na Internet] $2011 \mathrm{dez}$. [cited 2020 Apr. 20]; 2:94. Available from: https://www.frontiersin.org/articles/10.3389/fphys.2011.00094/full

11. Gill SR, Pop M, Deboy RT, Eckburg PB, Turnbaugh PJ, Samuel BS, Gordon JI, Relman DA, Fraser-Liggett $\mathrm{CM}$, Nelson KE. Metagenomic analysis of the human distal gut microbiome. Science [periódico na Internet] 2006 jun. [cited 2020 Mar. 27]; 312(5778):1355-1359. Available from: https://science.sciencemag.org/content/312/5778/1355.long

12. Farzi A, Fröhlich EE, Holzer P. Gut Microbiota and the Neuroendocrine System. Neurotherapeutics [periódico na internet] 2018 jan. [cited 2020 Mar. 30]; 15(1):5-22. Available from: https://www.ncbi.nlm.nih. gov/pmc/ articles/PMC5794709/
13. Rea K, Dinan TG, Cryan JF. The microbiome: a key regulator of stress and neuroinflammation. Neurobiol Stress [periódico na Internet] 2016 mar. [cited 2020 Apr. 1]; 4:23-33. Available from: https://www.ncbi. nlm.nih.gov/pmc/articles/PMC5146205/

14. Logan AC, Katzman M. Major depressive disorder: probiotics may be an adjuvant therapy. Med Hypotheses 2005; 64(3):533-538.

15. Karakula-Juchnowicz H, Rog J, Juchnowicz D, Łoniewski I, Skonieczna-Żydecka K, Krukow P, FutymaJedrzejewska M, Kaczmarczyk M. The study evaluating the effect of probiotic supplementation on the mental status, inflammation, and intestinal barrier in major depressive disorder patients using glutenfree or gluten-containing diet (SANGUT study): a 12-week, randomized, double-blind, and placebocontrolled clinical study protocol. Nutr $J$ [periódico na Internet] 2019 ago. [acessado 2020 abril 20]; 18(50). Available from: https://pubmed.ncbi.nlm.nih. gov/31472678/

16. Miller AH, Raison CL. The role of inflammation in depression: from evolutionary imperative to modern treatment target. Nat Rev Immunol [periódico na Internet] 2016 jan. [cited 2020 Apr. 4]; 16(1):22-34. Available from: https://pubmed.ncbi.nlm.nih.gov/ 26711676/

17. Dinan TG, Stanton C, Cryan JF. Psychobiotics: a novel class of psychotropic. Biol Psychiatry [periódico na Internet]. 2013 nov. [cited 2020 Mar. 15]; 74(10):720726. Available from: https://pubmed.ncbi.nlm.nih. gov/23759244/

18. Liberati A, Altman DG, Tetzlaff J, Mulrow C, Gøtzsche PC, Ioannidis JPA, Clarke M, Devereaux PJ, Kleijnen J, Moher D. The PRISMA statement for reporting systematic reviews and meta-analyses of studies that evaluate health care interventions: explanation and elaboration. BMJ [periódico na Internet]. 2009 jul. [cited 2020 Mar. 21]; 339:b2700. Available from: https://www.bmj.com/content/339/bmj.b2700.long

19. Chong HX, Yusoff NAA, Hor YY, Lew LC, Jaafar MH, Choi SB, Yusoff MSB, Wahid N, Abdullah MFIL, Zakaria N, Ong KL, Park YH, Liong MT. Lactobacillus plantarum DR7 alleviates stress and anxiety in adults: a randomised, double-blind, placebo-controlled study. Benef Microbes [periódico na Internet]. 2019 mar. [cited 2020 Apr. 16]; 10(4):355-373. Available from: https://pubmed.ncbi.nlm.nih.gov/30882244/

20. Tran N, Zhebrak M, Yacoub C, Pelletier J, Hawley D. The gut-brain relationship: Investigating the effect of multispecies probiotics on anxiety in a randomized placebo-controlled trial of healthy young adults. $J$ Affect Disord [periódico na internet] 2019 jun. [cited 2020 Apr. 16]; 252:271-277. Available from: https:// pubmed.ncbi.nlm.nih.gov/30991255/

21. Lew LC, Hor YY, Yusoff NAA, Choi SB, Yusoff MSB, Roslan NS, Ahmad A, Mohammad JAM, Abdullah MFIL, Zakaria N, Wahid N, Sun Z, Kwok LY, Zhang $\mathrm{H}$, Liong MT. Probiotic Lactobacillus plantarum P8 alleviated stress and anxiety while enhancing memory and cognition in stressed adults: A randomised, double-blind, placebo-controlled study. Clin Nutr [periódico na Internet] 2019 out. [cited 2020 Apr. 17]; 38(5):2053-2064. Available from: https://pubmed. ncbi.nlm.nih.gov/30266270/ 
22. Kelly JR, Allen AP, Temko A, Hutch W, Kennedy PJ Farid N, Murphy E, Boylan G, Bienenstock J, Cryan JF, Clarke G, Dinan TG. Lost in translation? The potential psychobiotic Lactobacillus rhamnosus (JB-1) fails to modulate stress or cognitive performance in healthy male subjects. Brain Behav Immun [periódico na Internet] 2017 mar. [cited 2020 Apr. 19]; 61:5059. Available from: https://pubmed.ncbi.nlm.nih. gov/27865949/

23. Romijn AR, Rucklidge JJ, Kuijer RG, Frampton C. A double-blind, randomized, placebo-controlled trial of Lactobacillus helveticus and Bifidobacterium longum for the symptoms of depression. Aust N Z J Psychiatry [periódico na Internet] 2017 ago. [cited 2020 Apr. 22]; 51(8):810-821. Available from: https://pubmed.ncbi. nlm.nih.gov/28068788/

24. Akkasheh G, Kashani-Poor Z, Tajabadi-Ebrahimi M, Jafari P, Akbari H, Taghizadeh M, Memarzadeh MR, Asemi Z, Esmaillzadeh A. Clinical and metabolic response to probiotic administration in patients with major depressive disorder: A randomized, double-blind, placebo-controlled trial. Nutrition [periódico na Internet] 2016 mar. [cited 2020 Apr. 23]; 32(3):315-320. Available from: https://pubmed.ncbi. nlm.nih.gov/26706022/

25. Mohammadi AA, Jazayeri S, Khosravi-Darani K, Solati Z, Mohammadpour N, Asemi Z, Adab Z, Djalali M, Tehrani-Doost M, Hosseini M, Eghtesadi S. The effects of probiotics on mental health and hypothalamic-pituitary-adrenal axis: A randomized, double-blind, placebo-controlled trial in petrochemical workers. Nutr Neurosci [periódico na Internet] 2016 nov. [cited 2020 Apr. 25]; 19(9):387-395. Available from: https://pubmed.ncbi.nlm.nih.gov/25879690/

26. Takada M, Nishida K, Kataoka-Kato A, Gondo Y, Ishikawa H, Suda K, Kawai M, Hoshi R, Watanabe O, Igarashi T, Kuwano Y, Miyazaki K, Rokutan K. Probiotic Lactobacillus casei strain Shirota relieves stress-associated symptoms by modulating the gut-brain interaction in human and animal models. Neurogastroenterol Motil [periódico na Internet] 2016 jul. [cited 2020 Apr. 21]; 28(7):1027-1036. Available from: https://pubmed.ncbi.nlm.nih.gov/26896291/

27. Steenbergen L, Sellaro R, van Hemert S, Bosch JA, Colzato LS. A randomized controlled trial to test the effect of multispecies probiotics on cognitive reactivity to sad mood Brain Behav Immun [periódico na Internet]. 2015 ago. [cited 2020 Apr. 28]; 48:258-264. Available from: https://pubmed.ncbi.nlm.nih.gov/25 862297/

28. Blier P, Ward HE, Tremblay P, Laberge L, Hébert C, Bergeron R. Combination of antidepressant medications from treatment initiation for major depressive disorder: a double-blind randomized study. $A m J$ Psychiatry [periódico na Internet] 2010 mar. [cited 2020 May. 1]; 167(3):281-288. Available from: https://ajp.psychiatryonline.org/doi/full/10.1176/appi. ajp.2009.09020186

29. O'Brien SM, Scott LV, Dinan TG. Cytokines: abnormalities in major depression and implications for pharmacological treatment. Hum Psychopharmacol [periódico na Internet] 2004 ago. [cited 2020 Mar. 28]; 19(6):397-403. Available from: https://onlinelibrary.wiley.com/doi/abs/10.1002/hup.609
30. Howren MB, Lamkin DM, Suls J. Associations of depression with C-reactive protein, IL-1, and IL-6: a meta-analysis. Psychosom Med 2009; 71(2):171-186.

31. Sarkar A, Lehto SM, Harty S, Dinan TG, Cryan JF, Burnet PWJ. Psychobiotics and the Manipulation of Bacteria-Gut-Brain Signals. Trends Neurosci [periódico na Internet] 2016 nov. [cited 2020 Apr. 17]; 39(11):763-781. Available from: https://pubmed.ncbi. nlm.nih.gov/27793434/

32. Yano JM, Yu K, Donaldson GP, Shastri GG, Ann P, Ma L, Nagler CR, Ismagilov RF, Mazmanian SK, Hsiao EY. Indigenous bacteria from the gut microbiota regulate host serotonin biosynthesis [published correction appears in Cell. 2015 Sep 24;163:258]. Cell [periódico na Internet] 2015 abr. [cited 2020 May 13]; 161(2):264-276. Available from: https://www.ncbi. nlm.nih.gov/pmc/articles/PMC4393509/

33. Banskota S, Ghia JE, Khan WI. Serotonin in the gut: Blessing or a curse. Biochimie [periódico na Internet] 2019 jun. [cited 2020 May 7]; 161:56-64. Available from: https://pubmed.ncbi.nlm.nih.gov/29909048/

34. Heinz A, Mann K, Weinberger DR, Goldman D. Serotonergic dysfunction, negative mood states, and response to alcohol. Alcohol Clin Exp Res 2001; 25(4):487-495.

35. Wrase J, Reimold M, Puls I, Kienast T, Heinz A. Serotonergic dysfunction: brain imaging and behavioral correlates. Cogn Affect Behav Neurosci [periódico na Internet] 2006 mar. [cited 2020 May 11]; 6(1):53-61. Available from: https://pubmed.ncbi.nlm.nih.gov/168 69229/

36. Hemarajata P, Versalovic J. Effects of probiotics on gut microbiota: mechanisms of intestinal immunomodulation and neuromodulation. Therap Adv Gastroenterol [periódico na Internet] 2013 jan. [cited 2020 Apr. 30]; 6(1):39-51. Available from: https://www.ncbi. nlm.nih.gov/pmc/articles/PMC3539293/

37. Lamprecht $M$, Frauwallner A. Exercise, intestinal barrier dysfunction and probiotic supplementation. Med Sport Sci [periódico na Internet] 2012 out. [cited 2020 May 3]; 59:47-56. Available from: https://www.karger. com/Article/Abstract/342169

38. Daliri EB-M, Lee BH. New perspectives on probiotics in health and disease. Food Sci Hum Wellness [periódico na Internet] 2015 jun. [cited 2020 May 23]; 4:56-65. Available from: https://core.ac.uk/download/ pdf/82355733.pdf

39. Huang R, Wang K, Hu J. Effect of Probiotics on Depression: A Systematic Review and Meta-Analysis of Randomized Controlled Trials. Nutrients [periódico na Internet] 2016 ago. [cited 2020 Apr. 9]; 8(8):483. Available from: https://www.mdpi.com/2072-6643 /8/8/483

Artigo apresentado em 19/06/2020

Aprovado em 26/06/2020

Versão final apresentada em 28/06/2020

Editores-chefes: Romeu Gomes, Antônio Augusto Moura da Silva 
